\title{
Refractive Dual Band Infrared Imager Optical Design
}

\author{
Ayatollah Karimzadeh \\ Optics and Laser Research Center, Amirkabir University of Technology, Tehran, Iran. \\ Corresponding Author Email: a.karimzadeh@aut.ac.ir \\ Received: Jul. 27, 2016, Revised: Oct. 25, 2016, Accepted: Dec. 6, 2016, Available Online: Aug. 8, 2017 \\ DOI: 10.18869 /acadpub.ijop.11.2.133
}

\begin{abstract}
Infrared imagers are important for reorganization and monitoring. This paper discusses the design of an infrared imager. Optical design in medium wavelength infrared (MWIR) and long wavelength infrared (LWIR) bands is different and needs distinct detectors and materials. Reflective systems are not suitable due to their small field of view (FOV) and vignetting. Refractive dual band optical system has been designed with considering both regions assessment and each band detector specifications.
\end{abstract}

KEYWORDS: Infrared imagers, dual band, medium wavelength infrared (MWIR), long wavelength infrared (LWIR), Thermal infrared.

\section{I.INTRODUCTION}

Infrared cameras are very important to identify and care. These cameras used to night vision, remote temperature measurement, in-line quality control, search and rescue.

The camera design in the mid-infrared camera medium wavelength infrared (MWIR) (3-5 $\mu \mathrm{m})$ is different with the thermal TIR (8-12 $\mu \mathrm{m})$ due to distinct in detectors and materials [1-4]. Reflective systems are not suitable due to their small field of view (FOV) and vignetting [1].

With production of new dual band detectors the produce and use of a dual band infrared optical system for long wavelength infrared (LWIR) and MWIR regions, is seems logical [5-11].

This paper presents the design of an infrared camera. Considering both regions requirements and detectors specifications, a common optical system is designed. Infrared materials' properties and their choosing method are described. The design of the dual band optical imaging system and its quality are analyzed and results are presented.

\section{OPTICAL SYSTEM DESIGN}

\section{A. Infrared Lens Materials}

The optical system has no reflective surface and formed from IR transparent elements. Germanium is a versatile infrared material commonly used in imaging systems, but in the mid infrared region, large dispersion of the germanium causes high chromatic aberration.

Abbe number $\mathrm{V \#}$ is the quantification for the dispersion, defined for the one region as follows:

$\mathrm{V} \#=\frac{\mathrm{n}_{\mathrm{C}}-1}{\mathrm{n}_{\mathrm{L}}-\mathrm{n}_{\mathrm{H}}}$

where $\mathrm{n}_{\mathrm{C}}, \mathrm{n}_{\mathrm{L}}$, and $\mathrm{n}_{\mathrm{H}}$ are respectively refractive indices in central, minimum and maximum wavelengths of the spectral region.

Whatever the Abbe number is smaller, dispersion becomes high, while low dispersion materials have higher Abbe number. For example, the germanium has large Abbe number about $4[1,2]$.

The silicon and germanium couple are used for correction of the chromatic aberration. Due to the low transitions of the silicon in thermal infrared area (Fig. 1), we could not use this couple for dual imaging system design. 


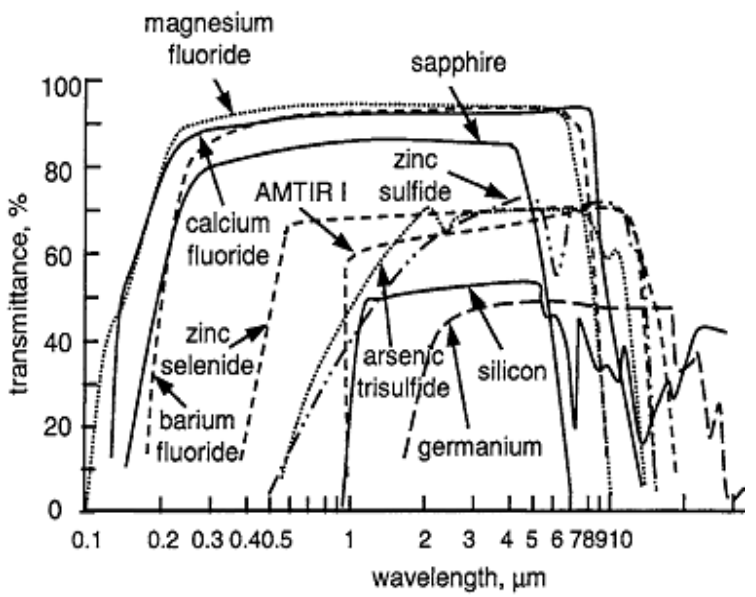

Fig. 1: Transmittance spectrum of infrared materials [1]

Table 1 shows the refraction index and Abbe number of the infrared materials in LWIR and MWIR regions.

Table 1. IR specifications of materials in (a) LWIR and (b) MWIR

\begin{tabular}{|c||lccc|c||}
\hline \hline \multirow{8}{*}{ a } & Region & $\mathrm{n}_{\mathrm{L}}$ & $\mathrm{n}_{\mathrm{H}}$ & $\mathrm{n}_{\mathrm{C}}$ & \multirow{2}{*}{ V\# } \\
\cline { 2 - 5 } & Length & $10 \mu \mathrm{m}$ & $8 \mu \mathrm{m}$ & $12 \mu \mathrm{m}$ & \\
\cline { 2 - 5 } & CAF2 & 1.300 & 1.350 & 1.231 & 2.5 \\
& ZnS & 2.202 & 2.223 & 2.176 & 25.5 \\
& ZnSe & 2.407 & 2.418 & 2.393 & 57.2 \\
& AMTR1 & 2.498 & 2.504 & 2.490 & 114.5 \\
& GERMA & 4.003 & 4.005 & 4.002 & 869.1 \\
& SILCN & 3.422 & 3.422 & 3.421 & 1818.9 \\
\hline \hline \multirow{6}{*}{$\mathrm{b}$} & Region & $\mathrm{n}_{\mathrm{L}}$ & $\mathrm{n}_{\mathrm{H}}$ & $\mathrm{n}_{\mathrm{C}}$ & \multirow{2}{*}{$\mathrm{V} \#$} \\
\cline { 2 - 4 } & Length & $4 \mu \mathrm{m}$ & $3.4 \mu \mathrm{m}$ & $5 \mu \mathrm{m}$ & \\
\hline & CAF2 & 1.410 & 1.415 & 1.399 & 25.8 \\
& ZnS & 2.252 & 2.255 & 2.246 & 136.5 \\
& ZnSe & 2.434 & 2.437 & 2.431 & 223.7 \\
& AMTR1 & 2.514 & 2.517 & 2.511 & 277.9 \\
& GERMA & 4.025 & 4.035 & 4.015 & 157.2 \\
& SILCN & 3.429 & 3.433 & 3.426 & 364.1 \\
\hline \hline
\end{tabular}

Due to the difference in lenses Abbe number, the chromatic aberration of the optical system can be corrected and optimized (Fig. 2) [1, 2].

\section{B. Infrared Detectors}

For maximum system sensitivity, most thermal imaging systems use cryogenically cooled detectors which operate at the liquid nitrogen temperature of $77 \mathrm{~K}$ or even lower.

This reduced temperature in cooled detectors is very important to reduce thermally induced noise to a level lower than that of the signal, from the scene being imaged.

These detectors are the high sensitive, especially when small "temperature targets" are observed over very long distances. Cooled detectors operate within the MWIR.

Uncooled infrared detectors do not require cryogenic cooling. One of the common designs is based on the microbolometer. Uncooled detectors operate within the LWIR region. This type of detector is generally much less expensive than the cooled type camera. One of the cost effective factors to consider is that cooled systems will be best to use beyond detection ranges of more than $5 \mathrm{~km}$. Another major contributing factor is the price of the lens of the complete system [2, 6, 7, and 12].

The $320 \times 256(40 \mu \mathrm{m}$ pixel size) focal plane array (FPA), mid-format 2-Color MWIR/LWIR Quantum Well Infrared Photodetectors (QWIP) are one of the dual band detectors, the specifications of which are given in Table 2.

Table 2. Specifications of $320 \times 256(40 \mu \mathrm{m}$ pixel size) FPA, mid-format 2-Color MWIR/LWIR QWIP detector [12]

\begin{tabular}{ll}
\hline Detector type & QWIP, MWIR/LWIR \\
\hline \hline Array size & $320 \times 256$ \\
Pixel size & $38 \mu \mathrm{m}$ \\
Pixel pitch & $40 \mu \mathrm{m}$ \\
Quantum efficiency & $15 \%$ for MWIR and 6\% for LWIR \\
\hline \hline
\end{tabular}

\section{Optical System Design}

Optical system is a $100 \mathrm{~mm} \mathrm{f} / 2$ imager with $320 \times 256(40 \mu \mathrm{m}$ pixel size $)$ FPA detector (Table 3).

Table 3. Imager specifications

\begin{tabular}{ll}
\hline \hline Parameter & Quantity \\
\hline \hline Focal length & $100 \mathrm{~mm}$ \\
Array size & $320 \times 256$ \\
Pixel size & $40 \mu \mathrm{m}$ \\
FOV & 9 degree \\
Spectral region & MWIR and LWIR \\
\hline
\end{tabular}

The design of optical system performed with OSLO optical design software. We choose a 
merit function for optimizing the optical system.

Due to the sensitivity curve of the infrared detectors, optical system design in 3.3-5 $\mu \mathrm{m}$ and 8 to $12 \mu \mathrm{m}$ ranges are selected, respectively for MWIR and LWIR regions. The zinc sulfide ( $\mathrm{ZnS})$ and zinc selenide (ZnSe) couple are used for designing the optical system. $\mathrm{ZnS}$ and $\mathrm{ZnSe}$ have the same behavior in MWIR and LWIR regions. Both of them are transparent in the mid-infrared and thermal infrared spectrum. Their distinct Abbe numbers are useful to chromatic aberration correction (Table 1).

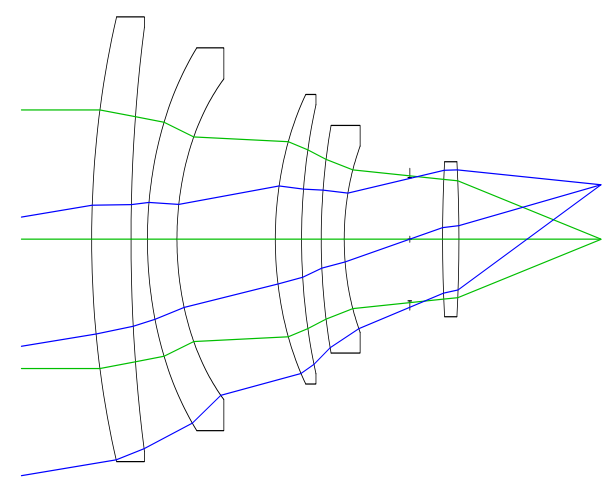

Fig. 3. Layout of designed optical system.

Designed optical system can be used separately for each of the MWIR and TIR spectral regions with the dependent detector (Table 4).

Table 4 Specification of the surfaces of the designed system of Fig. 3.

\begin{tabular}{ccccc}
\hline \hline Surface & $\begin{array}{c}\text { Radius } \\
(\mathrm{mm})\end{array}$ & $\begin{array}{c}\text { Thickness } \\
(\mathrm{mm})\end{array}$ & $\begin{array}{c}\text { Aperture } \\
\text { Radius }(\mathrm{mm})\end{array}$ & $\begin{array}{c}\text { Glass } \\
\text { type }\end{array}$ \\
\hline \hline 1 & 125.51 & 12 & 40 & ZnSe \\
2 & 208.51 & 5 & 37 & \\
3 & 53.00 & 9 & 35 & $\mathrm{ZnS}$ \\
4 & 40.74 & 30 & 28 & \\
5 & 46.95 & 8 & 25 & $\mathrm{ZnSe}$ \\
6 & 78.96 & 6 & 23 & \\
\hline 7 & 82.26 & 7 & 21 & $\mathrm{ZnS}$ \\
8 & - & 20 & 17 & \\
\hline 9 & 183.07 & 10 & 14 & ZnSe \\
10 & -177.15 & 5 & 14 & \\
\hline \hline
\end{tabular}

The vignetting of designed system for cooled detector with cold stop is acceptable. Although with increasing the lenses aperture or adding a relay lenses, we can completely omit the vignetting.
One lens can be used simultaneously for two MWIR and TIR detectors by the help of a beam splitter (Fig. 4). To minimize the beam splitter aberration effect, its thickness must be reduced or replace it by a cube beam splitter. However, the effect of beam splitter is negligible.

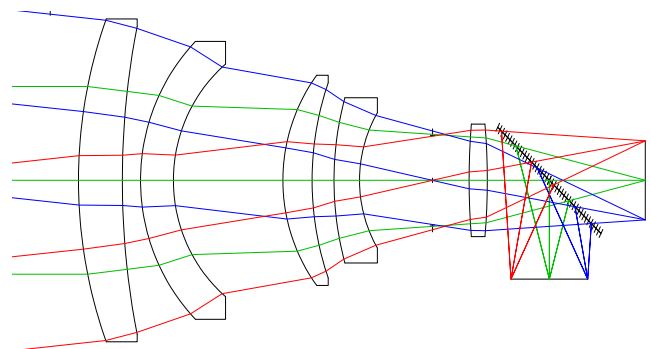

Fig. 4. Designed optical system with beam splitter

With using new dual band detectors, we could use this optical system simultaneously at LWIR and MWIR regions [3- 5].

\section{Optical System with Cold Stop}

If cooled detectors, or focal plane arrays (FPAs), are allowed to "see" any thermal energy other than the energy contained within the scene being viewed, then the sensitivity is reduced. In addition, if the magnitude of this nonscene energy changes or modulates over the field of view, then system has undesirable image anomalies. In order to achieve maximum sensitivity and avoid image anomalies, the IR FPA is cryogenically cooled and mounted into a thermally insulated "bottle," or dewar, assembly [1] (Fig. 5).

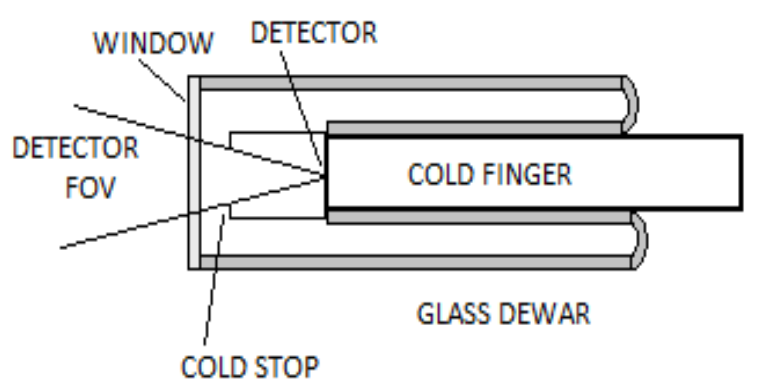

Fig. 5. Typical Detector Dewar Assembly

A baffle, called a cold shield or cold stop, is located inside the dewar, as shown in Fig. 5. An IR system is said to be $100 \%$ cold stop 
efficient if the detector can see or record energy only from the field of view [1].

We could decrease vignetting with adding relay section to optical system and reach to $100 \%$ cold stop efficiency.

The front objective lens is reimaged into the cold stop plane. Rays from various fields of view are shown all superimposed at image plane (Fig. 6).

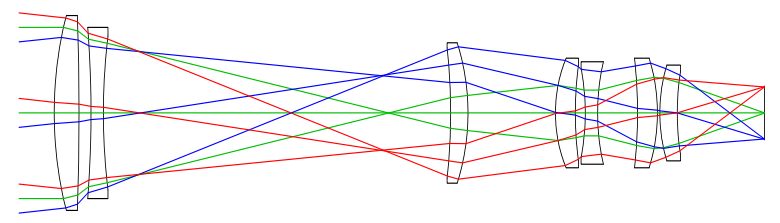

Fig. 6. Designed optical system with relay lens.

The end surface (surface 15) selected as aperture stop to have $100 \%$ cold stop efficient. This system diameter is smaller than the previous one, but its length is higher (Tables 4 and 5).

Table 5. Specifications of the surfaces of the optical system designed with cold stop of Fig. 6

\begin{tabular}{|c|c|c|c|c|}
\hline Surface & $\begin{array}{c}\text { Radius } \\
(\mathrm{mm})\end{array}$ & $\begin{array}{c}\text { Thickness } \\
(\mathrm{mm})\end{array}$ & $\begin{array}{c}\text { Aperture } \\
\text { Radius (mm) } \\
\end{array}$ & $\begin{array}{l}\text { Glass } \\
\text { type }\end{array}$ \\
\hline 1 & 85.94 & 10.0 & 28.5 & \multirow{2}{*}{$\mathrm{ZnSe}$} \\
\hline 2 & -603.0 & 4.83 & 28.5 & \\
\hline 3 & -232.93 & 5.0 & 25 & \multirow{2}{*}{$\mathrm{ZnS}$} \\
\hline 4 & 139.21 & 140 & 22.5 & \\
\hline 5 & -130.56 & 7 & 19 & \multirow{2}{*}{$\mathrm{ZnSe}$} \\
\hline 6 & -50.55 & 35.3 & 20.5 & \\
\hline 7 & 31.97 & 8 & 16 & \multirow{2}{*}{$\mathrm{ZnSe}$} \\
\hline 8 & 78.21 & 4 & 14 & \\
\hline 9 & -51.28 & 5 & 13 & \multirow{2}{*}{$\mathrm{ZnS}$} \\
\hline 10 & 50.12 & 16.1 & 15 & \\
\hline 11 & -103.37 & 8.0 & 16 & \multirow{2}{*}{$\mathrm{ZnSe}$} \\
\hline 12 & -40.00 & 1.0 & 16 & \\
\hline 13 & 36.51 & 7 & 14 & \multirow{3}{*}{$\mathrm{ZnS}$} \\
\hline $\begin{array}{l}14 \text { (aperture } \\
\text { stop) }\end{array}$ & 57.48 & 35.17 & 12 & \\
\hline 15 & -- & -- & 8 & \\
\hline
\end{tabular}

The relay section decreases the entrance ray and optical system diameter. The aperture stop of optical system is at the detector cold shield.

\section{E. Analysis of Optical Systems}

To optimize the uncooled optical system, the image spot size in two spectral regions has been considered as error function (merit function) and optimization is performed [13$15]$.

Figures 7(a) and 7(b) show the modulation transfer function (MTF) of the optical systems in the two channels. The MTF is acceptable at $40 \mathrm{cycle} / \mathrm{mm}$ frequency.

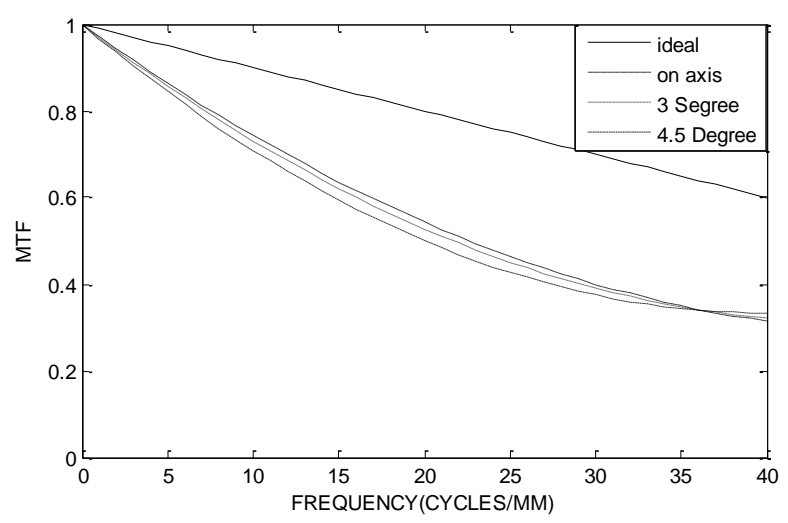

(a)

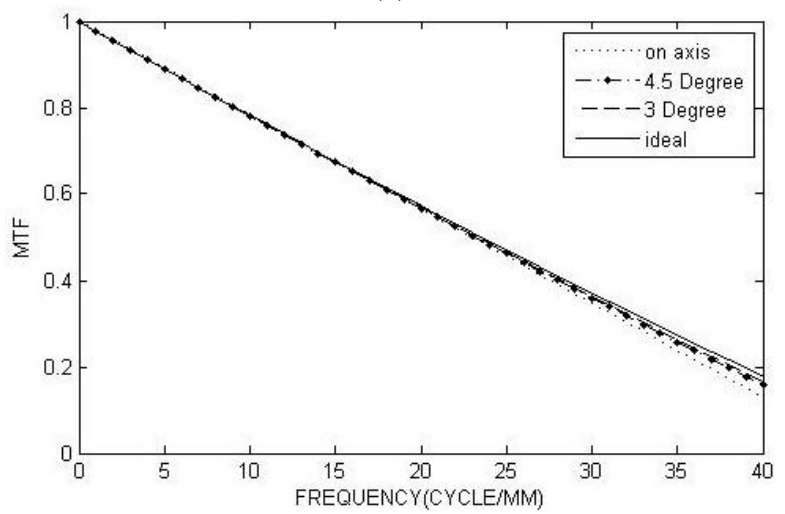

(b)

Fig. 7. Modulation transfer function of the optical system with relay lens for ideal case (diffraction limited), on axis (at $0^{\circ}$ field of view), 3 and 4.5 degrees fields of view for: a) 3.3 to $5 \mu \mathrm{m}$ and b) 8 to $12 \mu \mathrm{m}$.

Due to aperture stop location constraint of the cooled optical system (with relay lens), its optimizing is more difficult. The aperture stop must be located at the detector cooled stop. The optimized system diameter becomes smaller (Table 5) but its image quality decreases (Fig. 8).

Figures 8(a) and 8(b) show the MTFs of the optical systems for the two channels. The MTFs of the optical systems for MWIR and LWIR are higher than 0.2 at 30 cycle $/ \mathrm{mm}$ and $20 \mathrm{cycle} / \mathrm{mm}$ frequencies, respectively. 
The number of lenses at uncooled and cooled optical systems are 5 and 7 respectively (Figs. 3 and 6).

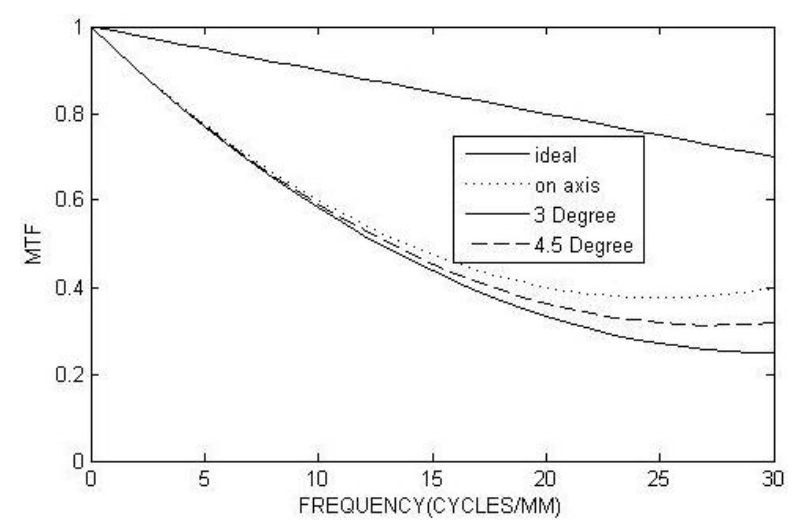

(a)

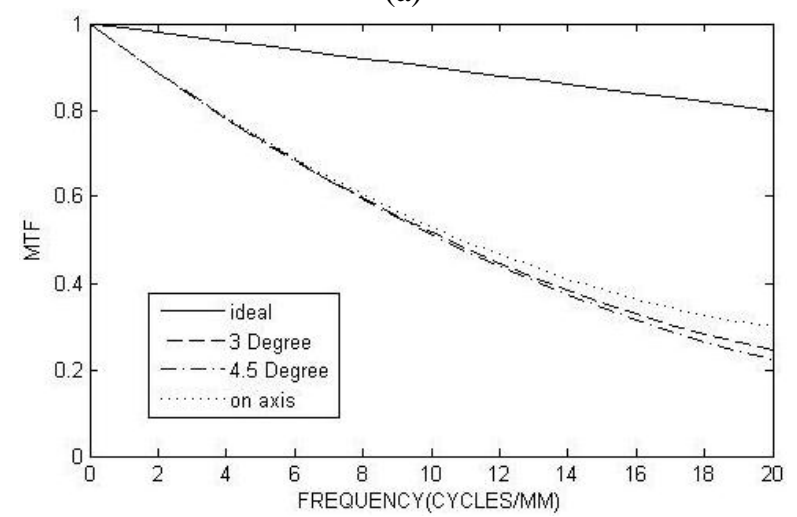

(b)

Fig. 8. Modulation transfer function of the optical system with relay lens for ideal case (diffraction limited), on axis (at $0^{\circ}$ field of view), 3 and 4.5 degrees fields of view of: a) 3.3 to $5 \mu \mathrm{m}$ and b) 8 to $12 \mu \mathrm{m}$ : a) 3.3 to $5 \mu \mathrm{m}$ and b) 8 to $12 \mu \mathrm{m}$.

The MTF of uncooled system (without cooled stop) is better (Figs. 7 and 8), but its vignetting and diameter are larger than cooled system.

\section{III.CONCLUSION}

With using appropriate materials with different dispersion witch has been transparent in both spectral regions of medium wavelength infrared (MWIR) and long wavelength infrared (LWIR), dual band infrared imaging system with uncooled and cooled detectors were designed and optimized. By comparison of uncooled and cooled infrared imaging systems, it has been shown that the image quality of uncooled imaging system is better.

\section{ACKNOWLEDGEMENTS}

The author is grateful to the Amir kabir University of Technology (AUT) research office for their support.

\section{REFERENCES}

[1] R.E. Fischer and B. Tadic-Galeb, Optical system design, McGraw-Hill, 2000.

[2] A. Karimzadeh and V. Karimzadeh, "Optical design of a dual band Infrared Imager," in 19th Iranian Conference on Optics and Photonics, Zahedan, 2013.

[3] M.J. Riedl, Optical design fundamentals for infrared systems, $2^{\text {nd }}$ Ed. SPIE Press, Washington USA, 2001.

[4] R. Khoei, "Optical design of a compact longrange thermal imaging camera in the 3-5 m wave band," J. Modern Opt. Vol. 58, pp. 619 624, 2011.

[5] M. Erdtmann, L.Zhang, and G. JinProc. "Uncooled dual-band MWIR/LWIR optical readout imager," SPIE, Vol. 6940, pp. 694012 (1-11), 2008.

[6] A. Rogalski, "Review Progress in focal plane array technologies," Prog. Quantum Electron. Vol. 36 , pp. 342-473, 2012

[7] O. Schreer, M.L. Sáenz, Ch. P. Eppermüller, and U. Schmidt, "Dual-band camera system with advanced image processing capability," Proc. SPIE Vol. 6542 , pp. 65421C (1-7), 2007

[8] H. Vogel , H. Schlemmer, and C. Zeiss Optronics GmbH (Germany)Proc. "Dual-band infrared camera," SPIE, Vol.5964, Detectors and Associated Signal Processing II, 59640S, 2005.

[9] A.G. McLean, J.-W. Ahn, R. Maingi, T.K. Gray, and A.L. Roquemore "A dual-band adaptor for infrared imaging," Rev. Sci. Instrum. Vol. 83, pp. 053706 (1-8), 2012.

[10] A. Rogalski, J. Antoszewski, and L. Faraone, "Third-generation infrared photodetector arrays," J. Appl. Phys, Vol. 105, pp. 091101 (1-44), 2009.

[11] S.D. Gunapala, S.B. Rafol, S.V. Bandara, J.K. Liu, J.M. Mumolo, A. Soibel, and D.Z. Ting, "Thermal imaging with novel infrared focal plane arrays and quantitative analysis of thermal imagery," Pasadena, CA: Jet 
Propulsion Laboratory, National Aeronautics and Space Administration, 2012.

[12] S.D. Gunapala, S.V. Bandara, J.K. Liu, C.J. Hill, S.B. Rafol, J.M. Mumolo, J.T. Trinh, M.Z. Tidrow, and P.D. LeVan, "Megapixel QWIP focal plane array and $320 \times 256$ pixel colocated mid-wave and long-wave dual-band QWIP focal plane array," SPIE, Quantum Sensing and Nanophotonic Devices II, Vol. 5732, pp. 295-308, 2005.

[13] A. Dikici, "A design study of a triple field of view 8-12 $\mathrm{mm}$ waveband airborne FLIR," Optics Laser Technol. Vol. 43, pp. 519-528, 2011

[14] R.R. Shannon, Mark, The Art and Science of Optical Design, ,Cambridge University Press, 2000

[15] W.J. Smith, Modern Lens Design, McGrawHill, 1992.

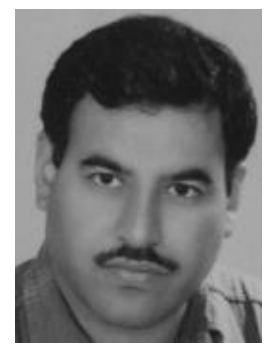

Ayatollah Karimzadeh received his BSc and MSc in Condensed matter Physics from Sharif University of Technology in 1995 and 1997, respectively and his $\mathrm{PhD}$ in Optics, from University of Isfahan, Iran, in 2008. He is currently Assistant professor of Physics at Amirkabir University of Technology, Tehran, Iran. His current research interests include: Optics and Photonics, Optical Biosensors, Imaging, Optical system design and test. 